\title{
Segmentation of SAR images using improved artificial bee colony algorithm and neutrosophic set
}

\author{
Kazim Hanbay ${ }^{\mathrm{a}, *}$, M. Fatih Talu ${ }^{\mathrm{b}}$ \\ a Informatics Department, Bingol University, 12000 Bingol, Turkey \\ b Computer Engineering Department, Inonu University, 44280 Malatya, Turkey
}

\section{A R T I C L E I N F O}

\section{Article history:}

Received in revised form 13 March 2014

Available online 24 April 2014

\section{Keywords:}

Synthetic aperture radar (SAR) images

Segmentation

Neutrosophic set

$\mathrm{I}-\mathrm{ABC}$ algorithm

\begin{abstract}
A B S T R A C T
This paper proposes a novel synthetic aperture radar (SAR) image segmentation algorithm based on the neutrosophic set (NS) and improved artificial bee colony (I-ABC) algorithm. In this algorithm, threshold value estimation is considered as a search procedure that searches for a proper value in a grayscale interval. Therefore, I-ABC optimization algorithm is presented to search for the optimal threshold value. In order to get an efficient and powerful fitness function for I-ABC algorithm, the input SAR image is transformed into the NS domain. Then, a neutrosophic $T$ and $I$ subset images are obtained. A co-occurrence matrix based on the neutrosophic $T$ and I subset images is constructed, and two-dimensional gray entropy function is described to serve as the fitness function of I-ABC algorithm. Finally, the optimal threshold value is quickly explored by the employed, onlookers and scouts bees in I-ABC algorithm. This paper contributes to SAR image segmentation in two aspects: (1) a hybrid model, having two different feature extraction methods, is proposed. (2) An optimal threshold value is automatically selected by maximizing the separability of the classes in gray level image by incorporating a simple and fast search strategy. The effectiveness of the proposed algorithm is demonstrated by application to real SAR images.
\end{abstract}

(c) 2014 Elsevier B.V. All rights reserved.

\section{Introduction}

Synthetic aperture radar (SAR) image segmentation is a significant part of SAR image analysis tasks. It ensures the entire structure of the image information and exposes useful information of SAR images. SAR images are used to define and interpret many objects in agriculture, urban design and military applications. In recent years, automated segmentation of SAR images has become a popular studying field [1-6]. Generally speaking, segmentation techniques of SAR images can be divided into two categories: feature-based algorithms [7-12] and model-based algorithms [13,14]. Additionally, region-based segmentation methods have also been used $[15,16]$. These methods reduce the computation complexity by working on regions instead of pixel neighborhood. Bai et al. [2] developed an edge detector which based on modified ratio and gradient of averages (MRGoA). According to them, a pixel is considered as an edge pixel only when both gradient and ratio of averages satisfy the desired threshold conditions. However, gradient is affected by the noise. Thus it is hard to get ideal results on fuzzy, discrete

\footnotetext{
* Corresponding author. Tel.: +90 5052937418.

E-mail addresses: kazimhanbay@gmail.com (K. Hanbay), fatihtalu@gmail.com (M.F. Talu).
}

and noisy object edges. Ranjani and Thiruvengadam [5] proposed entropy-based MRGoA model (EB-MRGoA) for SAR image segmentation. In [7], a powerful segmentation model using the existing ratio of exponential weighted averages (ROEWA) edge detector is developed. In this method, a manual threshold strategy is used for the segmentation of image. But, if the SAR image has more than one class, estimation of its threshold is difficult. By developing the ROEWA method, multi-level SAR image segmentation method (MROEWA) is proposed [8]. Yu et al. [10] proposed a segmentation method which based on context and feature information of image, and named as Context-based Hierarchical Unequal Merging for Synthetic aperture radar (SAR) Image Segmentation (CHUMSIS). This method uses super pixels as the operation units instead of pixels to reduce the influence of multiplicative speckle noises. Yu and Clausi [16] introduced region growing technique using semantics (IRGS and C-MLL) for SAR image segmentation.

Feature-based algorithms have the drawback of yielding images that are degraded by strong multiplicative speckle noise. Robust filtering is required to reduce the influence of the multiplicative speckle noise, which usually deteriorates the quality of segmentation of these algorithms. Model-based segmentation methods require mathematical foundation. They can extract or even enhance right object edges while removing speckle noise. These algorithms require an energy minimization process to find a satisfactory 
optimal solution for image [17]. For the energy minimization function, the split Bregman iteration is a proper choice [14]. Model-based methods have computational complexity. In general, model-based methods do not solve the issue of disconnected region segmentation and poor contrast in the SAR images.

This paper proposes a new segmentation method based on Improved Artificial Bee Colony (I-ABC) algorithm [18] and neutrosophic set $[19,20]$ for SAR images. I-ABC algorithm is used to optimize the two-dimensional gray entropy function and compute the global threshold value. The fitness functions of I-ABC algorithm based on two-dimensional gray entropy. The original $A B C$ algorithm is a Swarm Intelligence (SI) optimization technique such as Genetic Algorithm (GA), Ant Colony Optimization (ACO) and Artificial Fish Swarm (AFS) and based on the foraging behavior of honeybees $[21,22]$. These nature-inspired algorithms are successfully applied to numeric function optimization, filtering noisy transcranial Doppler signal, binary optimization, vehicle routing problem and image processing [23-26]. Neutrosophic set (NS) approach was suggested by Florentin Smarandache as a branch of philosophy dealing with the origin, nature and scope of neutralities, as well as their interactions with different ideational spectra [19]. In this theory, every event has not only a truth degree, but also a falsity degree and an indeterminacy degree that have to be evaluated independently from each other [19]. Guo and Cheng gave an example about reviewing paper to explain how the NS works [20].

The rest of the paper is organized as follows. In the next section, we comprehensively describe the neutrosophic set approach, the original ABC algorithm and I-ABC algorithm. Section "Proposed approach" presents the proposed hybrid segmentation algorithm. In section "Experimental results and discussions", experimental results and performance evaluation are presented in detailed. Noise-free images, noise images and well-known real SAR images used to evaluate the performance of the developed approach. In addition to this, the comparative evaluations of the segmentation results are discussed in terms of segmentation time, segmentation accuracy and convergence speed. Finally, some final remarks and conclusions are given in section "Conclusion".

\section{The I-ABC algorithm and neutrosophic set}

\section{The I-ABC algorithm}

The original artificial bee colony algorithm, which is natureinspired by the foraging behavior of real honey bees, was developed by D. Karaboga [21,22]. In this algorithm, artificial bees contain three groups bee: employed bee, onlooker bee and scout bee. Half of the colony consists of the employed bees, and the other half consists of the onlookers bees. Each food source represents corresponds to a possible solution to solve the optimization problem, and the nectar amount of each food source represents their quality (fitness) of the associated solution. For every food source, there is only one employed bee. So the number of the employed bees usually equals to the number of food sources. Whenever a food source has been abandoned by bees, the abandoned employed bee becomes a scout bee.

In the first place, the initial population $P$ and randomly distributed food source positions are generated. This process is performed as follows:

$x_{i j}=x_{\min , j}+\operatorname{rand}(0,1)\left(x_{\max , j}-x_{\min , j}\right)$

where the $x_{\min , j}$ and $x_{\max , j}$ are the lower and upper bounds in dimension $j$. The number of employed or onlooker bees is represented by $S N$. Every solution $x_{i}(i=1,2, \ldots, S N)$ is a $D$-dimensional vector, where $D$ represents the number of optimization parameters. After the initialization, the population of randomly produced solutions is depend on repeated cycles $C=1,2, \ldots, M C N$, where $M C N$ represents the number of maximum cycles. In first stage, employed bees search for a new better food source in neighborhood of a selected reference food source by modifying its location according to:

$v_{i j}=x_{i j}+\phi_{i j}\left(x_{i j}-x_{k j}\right)$

where $k \in\{1,2, \ldots, S N\}$ and $j \in\{1,2, \ldots, D\}$ are the randomly chosen index values: but $k$ must be different from $i$. Here $\phi_{i j}$ is a uniform random number in the range $[-1,1]$. $D$ denotes the problem variable dimensions. $x_{i j}$ and $x_{k j}$ is the location of the reference food source and the randomly selected food source in dimension $j$, respectively. If there is more nectar in new solution is than that in the reference one, the employed bees remember the new position and forget the old one. When all employed bees in the ABC algorithm have finished this food search process, they share the calculated nectar information and the position of the food sources with onlooker bees. Each of onlooker bees selects a food source completely by looking its probability value. When the nectar amount of the food source increases, the probability of being selected by onlookers likewise increases in the same way. The probability of choosing a food source $p_{i}$ could be calculated using Eq. (3):

$p_{i}=\frac{\text { fitness }_{i}}{\sum_{k=1}^{S N} \text { fitness }_{k}}$

where fitness ${ }_{i}$ denotes the fitness value of $i$ th food source. This update formulation used in the onlooker bee stage is the same as that in the employed bee stage. When the food source has been abandoned by employed bee, the employed bee becomes a scout bee. For the producing a new food position (solution), scout bee perform a random search using Eq. (1).

The food source exploitation is very important step in $A B C$. But the original $A B C$ algorithm has some inadequacies. Therefore, its convergence speed is also a problem in some applications. Li et al. [18] proposed a efficiency and fast $A B C$ method to improve the food search performance of $A B C$ algorithm. The improved $A B C$, which is called $I-A B C$, has a fast convergence speed. There are three significant differences between $A B C$ and I-ABC. I-ABC algorithm has the best-so-far solution, inertia weight and acceleration coefficients. These coefficients are providing a fast and efficient convergence. In $\mathrm{I}-\mathrm{ABC}$ algorithm, the modification forms of the employed bees and the onlooker ones can calculate as the following form:

$v_{i j}=x_{i j} W_{i j}+2\left(\phi_{i j}-0.5\right)\left(x_{i j}-x_{k j}\right) \Phi_{1}+\varphi_{i j}\left(x_{j}-x_{k j}\right) \Phi_{2}$

where $v_{i j}$ is the feasible solution that is an modified feasible solution depending on its previous solution $x_{i j} \cdot w_{i j}$ is the inertia weight coefficient which controls impacts of $x_{i j} \cdot x_{j}$ is the $j$ th parameter of the best-so-far solution, $\phi_{i j}$ and $\varphi_{i j}$ are random values in the range $[0,1] . \Phi_{1}$ and $\Phi_{2}$ are positive control parameters. Actually, I-ABC has worked with above-mentioned parameters. To overcome parameter selection problem, Li et al. [18] proposed a new formulation:

$w_{i j}=\Phi_{1}=\frac{1}{(1+\exp (-(\text { firness }(i)) / a p))}$

$\Phi_{2}=\left\{\begin{array}{cc}1 & \text { if a bee is employed one } \\ \frac{1}{(1+\exp (-(\text { fitness }(i)) / a p))} & \text { if a bee is onlooker one }\end{array}\right.$

where $a p$ is the fitness( 1 ) value in the first iteration

The most important advantages of I-ABC are to achieve a fast convergence speed and to find an optimal solution. Therefore, we employed the I-ABC algorithm instead of original ABC algorithm for SAR image segmentation. The experimental results tested on synthetic and real SAR images show that the I-ABC algorithm has perfect convergence speed and convergence characteristics 
comparing to original $\mathrm{ABC}$ algorithm and performs better than the state-of-art segmentation algorithm in well-known SAR images.

\section{Neutrosophic set}

An image is transformed into neutrosophic domain by using neutrosophic set formulations [27]. Thus, the neutrosophic image $P_{N S}$ is characterized by three membership sets $T, I$ and $F$. A pixel in the neutrosophic domain can be demonstrated as $P(t, i f)$ which means the pixel is $t \%$ true, $i \%$ indeterminate and $f \%$ false, where $t$ varies in $T, i$, varies in $I$, and $f$ varies in $F$, respectively. In classical set: $i=0, t$ and $f$ are either 0 or 100 . In fuzzy logic set: $i=0$, $0 \leq t, f \leq 100$. In neutrosophic set: $i=0,0 \leq t, i, f \leq 100$. A pixel $P(i, j)$ in the image domain is transformed into the neutrosophic domain, $P_{N S}(i, j)=\{T(i, j), I(i, j), F(i, j) . T(i, j), I(i, j)$ and $F(i, j)$ are the membership value belonging to true set, indeterminate set and false set, respectively.

$T(i, j)=\frac{\bar{g}(i, j)-\bar{g}_{\min }}{\bar{g}_{\max }-\bar{g}_{\min }}$

$\bar{g}(i, j)=\frac{1}{w \times w} \sum_{m=i-w / 2}^{i+w / 2} \sum_{n=j-w / 2}^{j+w / 2} g(m, n)$

$I(i, j)=\frac{\delta(i, j)-\delta_{\min }}{\delta_{\max }-\delta_{\min }}$

$\delta(i, j)=a b s(g(i, j)-\bar{g}(i, j))$

$F(i, j)=1-T(i, j)$

where $g^{-}(i, j)$ is local mean value of the input image. $\delta(i, j)$ is the absolute value of the difference between intensity $g(i, j)$ and its local mean value $\bar{g}(i, j)$ at $(i, j)$ pixel in the image.

The value of $I(i, j)$ is utilized to evaluate the indeterminacy degree of element $P_{N S}(i, j)$. For making $T$ and $F$ correlated with $I$, the changes in $T$ and $F$ influence the pixel distribution of element in $I$ and the entropy of $I$. $\alpha$-Mean and $\beta$-enhancement operations, are utilized to decrease the set indeterminacy in NS image.

For a gray level image $I$, a mean operation is defined as:

$\bar{l}(i, j)=\frac{1}{w \times w} \sum_{m=i-w / 2}^{i+w / 2} \sum_{n=j-w / 2}^{j+w / 2} l(m, n)$

Using the a parameter, an $\alpha$-mean operation $\bar{P}_{N S}(\alpha)$ is described to calculate the mean value between the pixel neighbors:

$\bar{P}_{N S}(\alpha)=P(\bar{T}(\alpha), \bar{I}(\alpha), \bar{F}(\alpha))$

$\bar{T}(\alpha)=\left\{\begin{array}{ll}T, & I<\alpha \\ \bar{T} \alpha, & I \geq \alpha\end{array}\right\}$

$\bar{T}_{\alpha}(i, j)=\frac{1}{w \times w} \sum_{m=i-w / 2}^{i+w / 2} \sum_{n=j-w / 2}^{j+w / 2} T(m, n)$

$\bar{F}(\alpha)=\left\{\begin{array}{ll}F, & I<\alpha \\ \bar{F}, & I \geq \alpha\end{array}\right\}$

$\bar{F}_{\alpha}(i, j)=\frac{1}{w \times w} \sum_{m=i-w / 2}^{i+w / 2} \sum_{n=j-w / 2}^{j+w / 2} F(m, n)$

$\bar{I}_{\alpha}(i, j)=\frac{\bar{\delta}_{T}(i, j)-\bar{\delta}_{T} \min }{\bar{\delta}_{T} \max -\bar{\delta}_{T} \min }$

$\bar{\delta}_{T}(i, j)=a b s(\bar{T}(i, j)-\overline{\bar{T}}(i, j))$
$\overline{\bar{T}}(i, j)=\frac{1}{w \times w} \sum_{m=i-w / 2}^{i+w / 2} \sum_{n=j-w / 2}^{j+w / 2} \bar{T}(m, n)$

where $\bar{\delta}_{T}(i, j)$ is the absolute value of the difference between the mean intensity $\bar{T}(i, j)$ and its mean value $\overline{\bar{T}}(i, j)$ after the $\alpha$-mean operation. After the $\alpha$-mean operation, the entropy of the indeterminate subset $I$ is increased. Consequently, $I$ has more a uniform element distribution.

Similar to fuzzy set, $\beta$-enhancement operation, $P_{N S}^{\prime}(\beta)$ is computed as:

$P_{N S}^{\prime}(\beta)=P\left(T^{\prime}(\beta), I^{\prime}(\beta), F^{\prime}(\beta)\right)$

$T^{\prime}(\beta)=\left\{\begin{array}{ll}T, & I \triangleleft \beta \\ T_{A}^{\prime} & I \geq \beta\end{array}\right\}$

$T_{\beta}^{\prime}(i, j)=\left\{\begin{array}{ll}2 T^{2}(i, j), & T(i, j) \leq 0.5 \\ 1-2(1-T(i, j))^{2}, & T(i, j)>0.5\end{array}\right\}$

$F^{\prime}(\beta)=\left\{\begin{array}{ll}F, & I<\beta \\ F_{\lambda}^{\prime}, & I \geq \beta\end{array}\right\}$

$F_{\beta}^{\prime}(i, j)=\left\{\begin{array}{ll}2 F^{2}(i, j), & F(i, j) \leq 0.5 \\ 1-2(1-F(i, j))^{2}, & F(i, j)>0.5\end{array}\right\}$

$I_{\beta}^{\prime}(i, j)=\frac{\delta_{T}^{\prime}(i, j)-\delta_{T}^{\prime} \min }{\delta_{T}^{\prime} \max -\delta^{\prime}{ }_{T} \min }$

$\delta_{T}^{\prime}(i, j)=a b s\left(T^{\prime}(i, j)-\overline{T^{\prime}}(i, j)\right)$

$\bar{T}^{\prime}(i, j)=\frac{1}{w \times w} \sum_{m=i-w / 2}^{i+w / 2} \sum_{n=j-w / 2}^{j+w / 2} T^{\prime}(m, n)$

where $\delta_{T}^{\prime}(i, j)$ is the absolute value of difference between intensity $T^{\prime}(i, j)$ and its local mean value $\bar{T}^{\prime}(i, j)$ at $(i, j)$ after the $\beta$-enhancement operation. As a result of $\beta$-enhancement operation, the $T$ set becomes more suitable for segmentation and entropic thresholding. Since membership set $T$ has high contrast, the background and objects in the image are correctly distinguished.

In the previous studies, $\alpha$ and $\beta$ parameters were selected as a constant value: 0.85 , which significantly affect the segmentation quality. Sengur and Guo have proposed a method to overcome the above-mentioned parameter selection problem [27]. According to them, $\alpha$ and $\beta$ parameters are selected adaptively considering the characteristic of the processed images. These parameters can be expressed as:

$E n I=-\sum_{i=1}^{h} \sum_{j=1}^{w} p(i, j) \log _{2} p(i, j)$

$E n_{\max }=-\log _{2} \frac{1}{h w}$

$\delta=\delta_{\min }+\frac{\left(\delta_{\max }-\delta_{\min }\right)\left(E n I-E n_{\min }\right)}{\left(E n_{\max }-E n_{\min }\right)}$

$\beta=1-\delta$

where $h$ and $w$ are the height and width of the neutrosophic image. Here, $E n_{\min }=0, \alpha_{\min }=0.01$ and $\alpha_{a x}=0.1$ are determined by our SAR image segmentation experiments.

\section{Proposed approach}

In this section, we propose a new segmentation algorithm, which is applied to segmentation of SAR images. The new algorithm uses the I-ABC algorithm and the neutrosophic set. In recent 
years, neutrosophic set has been applied to different computer vision problems such as image thresholding, image segmentation, image denoising and classification applications [28-30]. The neutrosophic set can suppress the edge fuzzy and provide accurate edge location. Consequently in the proposed algorithm, the local feature of edge strength is incorporated into the two-dimensional entropy function. In addition to this, methods based on the Artificial Bee Colony algorithm and neutrosophic set derived from entropic thresholding model have demonstrated incredible performance in various image segmentation applications [23,29,31-33]. These types of hybrid methods have the superiority of optimal threshold value calculation while simultaneously denoising the image.

The proposed method is composed of two main steps: feature extraction process and two-dimensional gray entropy-based thresholding. We use the neutrosophic set method to obtain significant image features. Instead of neutrosophic $T$, $I$ and $F$ subsets, we propose to use only neutrosophic $T$ and $I$ subsets to construct the co-occurrence matrix. Co-occurrence matrix provides the space relationship between each pixel and its adjacent pixels. This method is suitable for segmentation of SAR images degraded by strong speckle noise. Image features obtained from neutrosophic domain are used to achieve robustness of co-occurrence matrix. In the first step, the gray scale image is obtained from the RGB color input SAR image. Then, the all pixels $\mathrm{P}(i, j)$ in the image domain are transformed into the neutrosophic domain using Eqs. (7)-(11). The indeterminacy of the NS image $P_{N S}$ is decreased by using the $\alpha$-mean and $\beta$-enhancement operations on subset $T$ according to Eqs. (13)-(28). The values of $\alpha$ and $\beta$ parameters are computed automatically using Eqs. (29)-(32). $T$ and $I$ subset images are normalized to have unit standard deviations. Normalization process helps to advance segmentation performance. Since $T$ and $I$ subsets are more convenient for segmentation, $F$ subset have been ignored. In the next step, the elements in subset $T$ and $I$ are combined as the input for the construct a $L \times L^{\prime}$ neutrosophic $T-I$ co-occurrence matrix $C=\left[c_{i j}\right]_{L \times L^{\prime}}$. While $c_{i j}$ shows the number of pixel pairs satisfying $T(m, n)=i$ and $I(m, n)=j, L=L^{\prime}=256$ shows the gray levels number in the $T$ and $I$ subset images. The priori probability $p_{i j}$ of a pixel pair $(i, j)$ in the co-occurrence matrix is explained by the total number of occurrences of the pixel pair. Therefore, $p_{i j}$ can be written as [33]:

$p_{i j}=\frac{c_{i j}}{\sum_{i=0}^{L-1} \sum_{j=0}^{L^{\prime}-1} c_{i j}}$

The two-dimensional threshold vector $(s, t)$ can partition the cooccurrence matrix into four quadrant regions. These four quadrants are shown Fig. 1.

If an image has a uniform gray level distribution, it may not contain different objects or edges. Therefore, $T$ and $I$ subset images have a similar neutrosophic behaviors. While the $T$ subset consists of smooth image information, the $I$ subset consists of both edge information and texture information. In Fig. $1 \mathrm{~A}$ and $\mathrm{D}$ are present object and background, respectively.

Since $A$ and $C$ quadrants have even the same neutrosophic values, they are ignored in the entropy calculation. But, B represents edge and texture feature in object regions, and D represents edge and texture feature in background regions. For a gray level image, the entropy is utilized to evaluate the distribution of the gray levels in a scene [34]. If the entropy is big, the intensities have equal probability and their distributions are uniform. If the entropy is small, the intensity distribution is nonuniform.

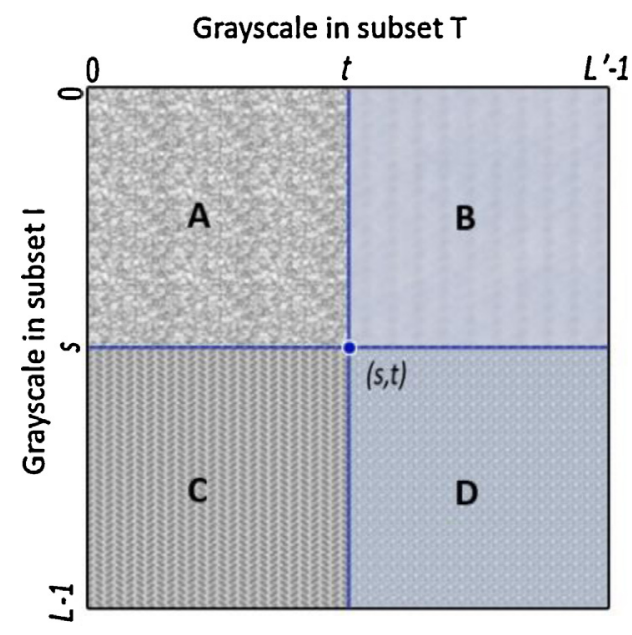

Fig. 1. Quadrants of neutrosophic $T-I$ subset co-occurrence matrix.

Shannon conditional entropies of these quadrants can be calculated as [35]:

$H\left(\frac{\text { edge }}{\text { object }}\right)=H\left(\frac{E}{O}\right)=-\sum_{i=0}^{s} \sum_{j=t+1}^{L^{\prime}} p_{i j}^{B} \log _{2} p_{i j}^{B}$
$H\left(\frac{\text { edge }}{\text { background }}\right)=H\left(\frac{E}{B}\right)=-\sum_{i=s+1}^{L-1} \sum_{j=t+1}^{L^{\prime}-1} p_{i j}^{D} \log _{2} p_{i j}^{D}$

Hence, the total second-order Shannon conditional entropy of the two images can be calculated as:

$H_{(c)}^{T}(s, t)=\frac{H(E / O)+H(E / B)}{2}$

The maximum of $H_{(c)}^{T}(s, t)$ value gives the optimal threshold vector for object and background segmentation. In this stage of the proposed algorithm, the entropic threshold calculation process is employed and objective function is defined in the range $\left[(0 \leq s \leq L-1)\right.$ and $\left(0 \leq t \leq L^{\prime}-1\right)$ as follows:

$\operatorname{Optimal}(s, t)=\operatorname{Arg}\left\{\max H_{(c)}^{T}(s, t)\right\}$

In this case, the process includes finding a threshold vector $(s, t)$ which maximizes the $H_{(c)}^{T}(s, t)$. According to the two-dimensional gray entropy theory, when the difference between backgrounds and objects reaches to the biggest value, $(s, t)$ is the best position to segment $T$ and $I$. To calculate the best position of $(s, t)$, we use the I$\mathrm{ABC}$ algorithm instead of original $\mathrm{ABC}$ algorithm. The pseudo-code of the I-ABC algorithm is given below.

\section{Algorithm 1: I-ABC}
1. Initialize population $P$
2. repeat
3. while Termination condition is not satisfied do
4. Employed bee Generates $v_{i}$ for employed bees by (2)
5. Evaluate and apply the greedy selection process.
6. Onlookers Calculate $p_{i}$ for $x_{i}$ by (3)
7. Generates $v_{i}$ for the onlookers from $x_{i}$ based on $p_{i}$.
8. Evaluate and apply the greedy selection process.
9. Scout Determine the abandoned $x_{i}$, if exists, update it by (1).
10. Update the best solution acquired so far
11. end while
12. Output Global optimum value $Q_{\mathrm{s}}$
13. until all the optimum gained 
Two-dimensional gray entropy function gives substantially more promising results in SAR image segmentation. The segmentation quality of our method results from the two-dimensional gray entropy function, for the fact that speckle noise almost completely disappears, and most useful informations about edge and texture are preserved. Namely, two-dimensional gray entropy function obtained from the co-occurrence matrix is used as the fitness function of I-ABC algorithm. Therefore, we utilized used Eq. (4) instead of Eq. (2) in order to produce a candidate food position. Here, each bee in I-ABC algorithm is two-dimensional, meaning a pair of gray numbers $(s, t)$. The population size is the number of possible threshold positions searched in parallel. The position of a food source represents a possible threshold value. The nectar amount of a food source in I-ABC algorithm corresponds to the quality of a segmented SAR image evaluated using Eq. (37). lows:

The proposed segmentation algorithm is summarized as fol-

Step 1: Convert the input SAR image from RGB color space to gray level domain.

Step 2: Transform the gray level SAR image into NS domain using Eqs. (7)-(11), which are represented as T, I, F.

Step 3: $\alpha$ and $\beta$ parameters are obtained using Eqs. (29)-(32) and perform the $\alpha$-mean and $\beta$-enhancement operations on the $T$ and I subsets using Eqs. (13)-(28).

Step 4: Normalize the neutrosophic $T$ subset image and neutrosophic I subset image.

Step 5: Using the neutrosophic $T$ and $I$ subset images, construct a $256 \times 256$ co-occurrence matrix $C$ to obtain the two-dimensional gray entropy.

Step 6: Treat the two-dimensional gray entropy function as the fitness function of I-ABC algorithm, and adjust the all control parameters in I-ABC algorithm.

Step 7: The best bee gradually finds an optimal threshold vector $H_{(c)}^{T}(s, t)$ through collaboration and information-sharing of multiple cycles of employed, onlooker and scout bees.

Step 8: Segment the neutrosophic T subset image with the optimal threshold $s$ and get the final segmented SAR image.

\section{Experimental results and discussions}

In this section, synthetic and real SAR images are utilized to illustrate the performance of the proposed algorithm. Firstly, we compared our algorithm with nature-inspired SAR image segmentation methods which based on Artificial Bee Colony (ABC, Genetic Algorithm (GA) and Artificial Fish Swarm (AFS) algorithm. Secondly, our proposed method is compared with well-known state-of-the-art machine learning approaches such as contextbased, edge-based and region based. In this experiment, for I-ABC algorithm, the population size is 20 , the number of iterations is 30 , and the limit times for abandonment is 10 , the lower and upper bounds are 0 and 255 respectively. Our algorithm is coded by Matlab R2011b. All the experiments in this section are run in a release version of our code on an Intel(R) Core(TM) i5-2500 CPU, 2 GB RAM personal computer.

\section{Comparison of nature-inspired algorithms}

In this section, our SAR image segmentation algorithm is tested and compared with the GA and AFS methods in terms of segmentation result, convergence speed and CPU time. Since the segmentation algorithm proposed by Ma et al. [23] using the ABC algorithm in SAR image segmentation, we select it in the comparison. The other two nature-inspired algorithms include the following: the GA segmentation [24] and the AFS segmentation
Table 1

Comparison results on segmentation time in case of Fig. 2(a).

\begin{tabular}{|c|c|c|c|c|}
\hline Method & SI schema & Fitness function & $\begin{array}{l}\text { Threshold } \\
\text { value }\end{array}$ & Time (s) \\
\hline $\begin{array}{l}\text { The proposed } \\
\text { method }\end{array}$ & $\mathrm{I}-\mathrm{ABC}$ & $\begin{array}{l}\text { Improved } \\
\text { two-dimensional } \\
\text { gray entropy }\end{array}$ & 204 & 2.275 \\
\hline The method [23] & $\mathrm{ABC}$ & $\begin{array}{l}\text { Improved } \\
\text { two-dimensional } \\
\text { gray entropy }\end{array}$ & 205 & 4.451 \\
\hline The method [24] & GA & $\begin{array}{l}\text { Two-dimensional } \\
\text { entropy }\end{array}$ & 207 & 12.428 \\
\hline The method [25] & AFS & $\begin{array}{l}\text { Two-dimensional } \\
\text { Otsu }\end{array}$ & 187 & 11.276 \\
\hline
\end{tabular}

[25], which is briefly mentioned in the introduction. For the GA and AFS algorithm parameters, we used default values as described in [23].

Here we choose four synthetic and real SAR images to test the performance and high convergence speed of the proposed algorithm. The synthetic and original SAR images are given in the first row, proposed method's segmentation results are given in the second row, the $A B C$ method's segmentation results are given in the third row, the GA method's segmentation results are given in the fourth row and the AFS based method's segmentation results are given in the fifth row of Fig. 2, respectively. As we see, our proposed method and the $\mathrm{ABC}$ method obtained correct object regions. It should be noted that the $A B C$ method [23] can extract object region. But, the $A B C$ method cannot segment synthetic image in third column (Fig. 2(k)). The proposed method gets clear and wellconnected object boundaries for this image. So the proposed hybrid method based on the neutrosophic set and two-dimensional gray entropy model could not only remove the disruptive effect of multiplicative speckle noise well but also could obtain a cleaner object region than the method based on GA and AFS model. Since GA and AFS methods suffer from multiplicative speckle noise, these methods cannot give a satisfactory segmentation result in region or edge.

(a) Comparison of segmentation time

Compared to $\mathrm{I}-\mathrm{ABC}$ and $\mathrm{ABC}$ algorithm in terms of computation time, the I-ABC shows better performance than $A B C$. To compare the segmenting time spent in Fig. 2, experimental results are shown in Tables $1-4$. The results of $A B C$, GA and AFS are obtained by applying the original codes.

(b) Comparison of convergence speed

We analyze and compare the convergence speed among our method, ABC, GA and AFS. As we see, Fig. 3(a) shows that I-ABC algorithm converges at the 7th iteration and finds the optimal threshold value. Fig. 3(b) shows that original $A B C$ algorithm converges at the 12th iteration. Fig. 3(c) shows the GA do not converge yet at the 30th iteration, so it certainly cannot find

Table 2

Comparison results on segmentation time in case of Fig. 2(b).

\begin{tabular}{|c|c|c|c|c|}
\hline Method & SI schema & Fitness function & $\begin{array}{l}\text { Threshold } \\
\text { value }\end{array}$ & Time (s) \\
\hline $\begin{array}{l}\text { The proposed } \\
\text { method }\end{array}$ & $\mathrm{I}-\mathrm{ABC}$ & $\begin{array}{l}\text { Improved } \\
\text { two-dimensional } \\
\text { gray entropy }\end{array}$ & 200 & 2.279 \\
\hline The method [23] & $\mathrm{ABC}$ & $\begin{array}{l}\text { Improved } \\
\text { two-dimensional } \\
\text { gray entropy }\end{array}$ & 204 & 4.438 \\
\hline The method [24] & GA & $\begin{array}{l}\text { Two-dimensional } \\
\text { entropy }\end{array}$ & 163 & 13.413 \\
\hline The method [25] & AFS & $\begin{array}{l}\text { Two-dimensional } \\
\text { Otsu }\end{array}$ & 162 & 10.498 \\
\hline
\end{tabular}




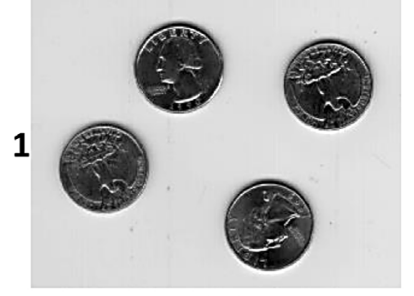

(a)

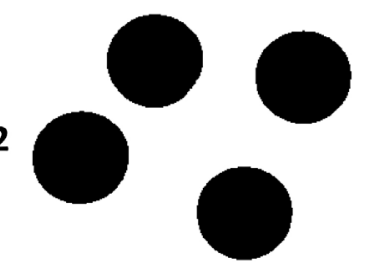

(e)

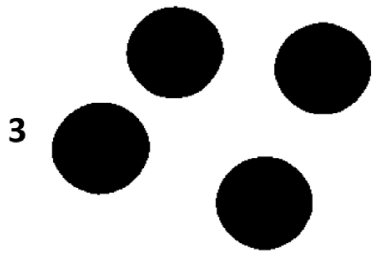

(i)

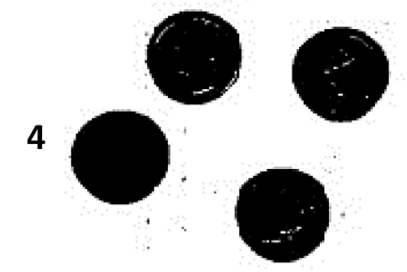

(m)

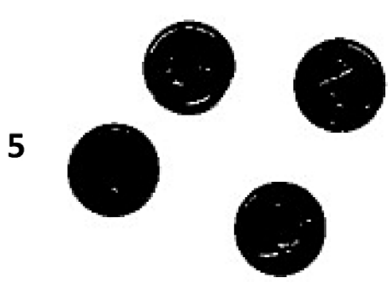

(r)

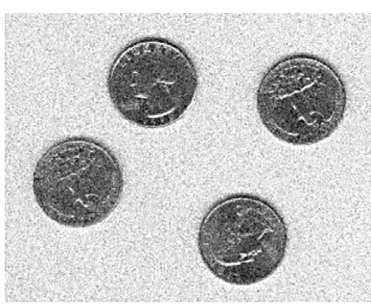

(b)

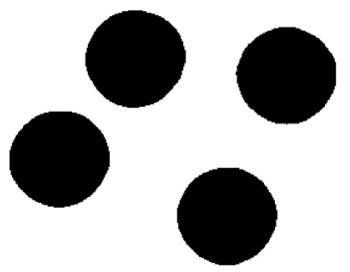

(f)

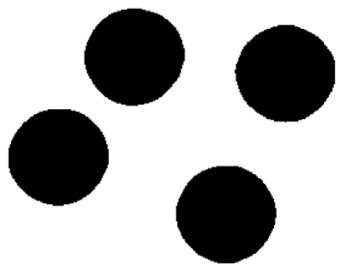

(j)

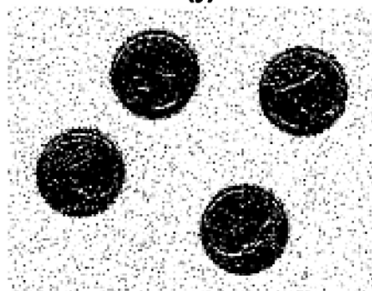

(n)

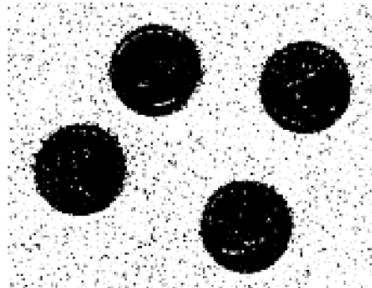

(s)

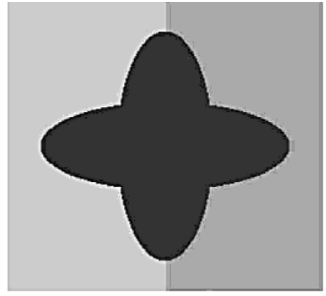

(c)

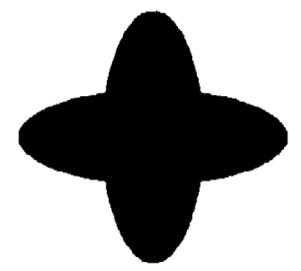

(g)

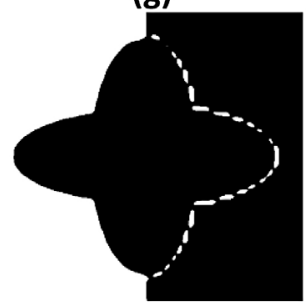

(k)

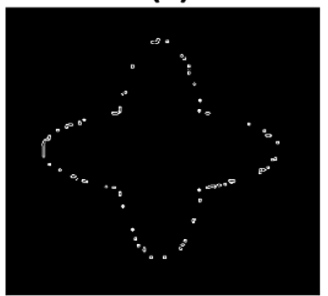

(o)

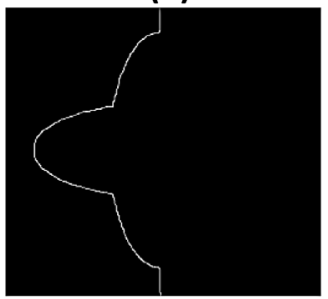

(t)

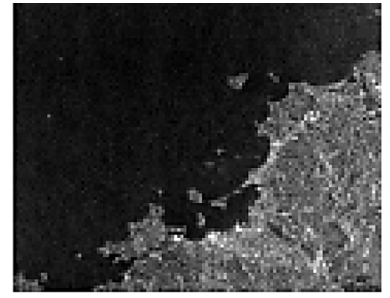

(d)

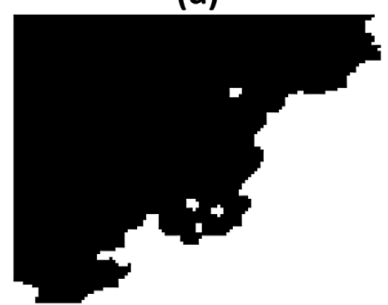

(h)

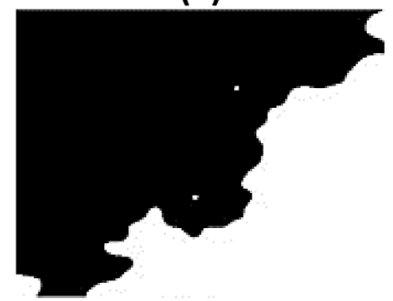

(I)

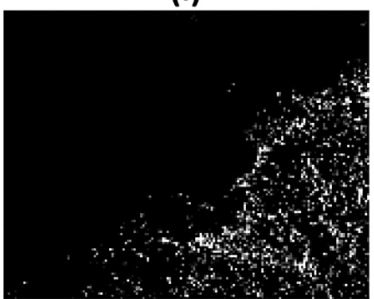

(p)

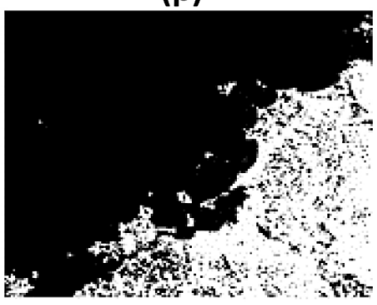

(u)

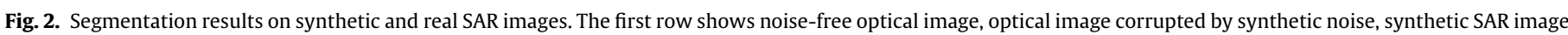
and original SAR image, respectively. Segmentation results are displayed on rows 2, 3, 4, 5 for the proposed method, ABC method [23], GA [24] and AFS [25], respectively.

Table 3

Comparison results on segmentation time in case of Fig. 2(c).

\begin{tabular}{|c|c|c|c|c|}
\hline Method & SI schema & Fitness function & $\begin{array}{l}\text { Threshold } \\
\text { value }\end{array}$ & Time (s) \\
\hline $\begin{array}{l}\text { The proposed } \\
\text { method }\end{array}$ & $\mathrm{I}-\mathrm{ABC}$ & $\begin{array}{l}\text { Improved } \\
\text { two-dimensional } \\
\text { gray entropy }\end{array}$ & 152 & 2.167 \\
\hline The method [23] & $\mathrm{ABC}$ & $\begin{array}{l}\text { Improved } \\
\text { two-dimensional } \\
\text { gray entropy }\end{array}$ & 232 & 2.762 \\
\hline The method [24] & GA & $\begin{array}{l}\text { Two-dimensional } \\
\text { entropy }\end{array}$ & 249 & 15.42 \\
\hline The method [25] & AFS & $\begin{array}{l}\text { Two-dimensional } \\
\text { Otsu }\end{array}$ & 238 & 13.71 \\
\hline
\end{tabular}

Table 4

Comparison results on segmentation time in case of Fig. 2(d).

\begin{tabular}{|c|c|c|c|c|}
\hline Method & SI schema & Fitness function & $\begin{array}{l}\text { Threshold } \\
\text { value }\end{array}$ & Time (s) \\
\hline $\begin{array}{l}\text { The proposed } \\
\text { method }\end{array}$ & $\mathrm{I}-\mathrm{ABC}$ & $\begin{array}{l}\text { Improved } \\
\text { two-dimensional } \\
\text { gray entropy }\end{array}$ & 75 & 2.389 \\
\hline The method [23] & $\mathrm{ABC}$ & $\begin{array}{l}\text { Improved } \\
\text { two-dimensional } \\
\text { gray entropy }\end{array}$ & 95 & 3.824 \\
\hline The method [24] & GA & $\begin{array}{l}\text { Two-dimensional } \\
\text { entropy }\end{array}$ & 131 & 11.648 \\
\hline The method [25] & AFS & $\begin{array}{l}\text { Two-dimensional } \\
\text { Otsu }\end{array}$ & 62 & 5.492 \\
\hline
\end{tabular}




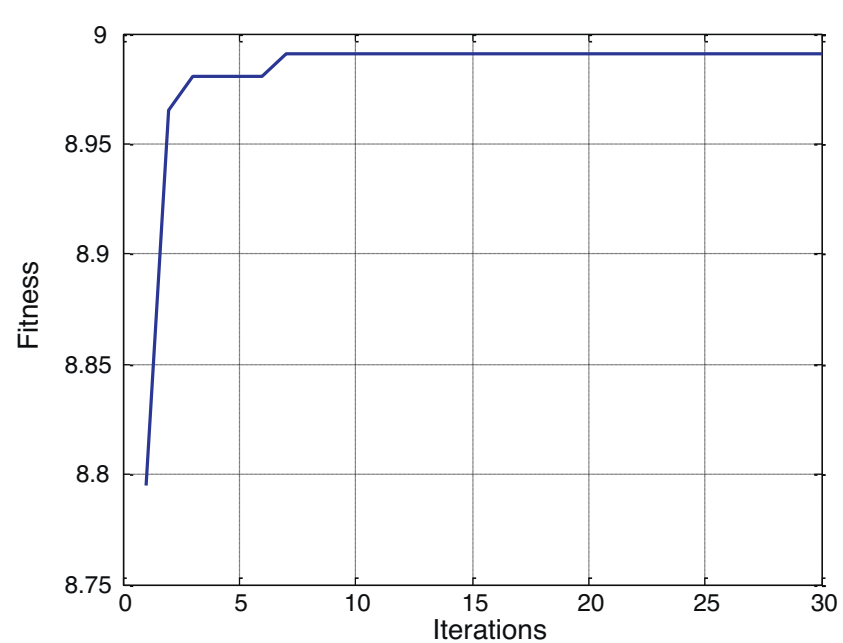

(a)

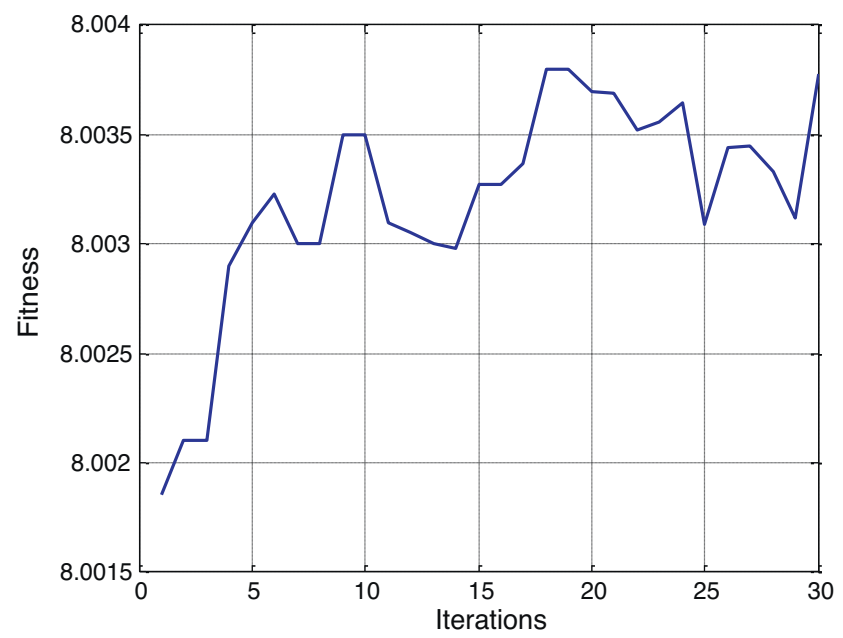

(c)

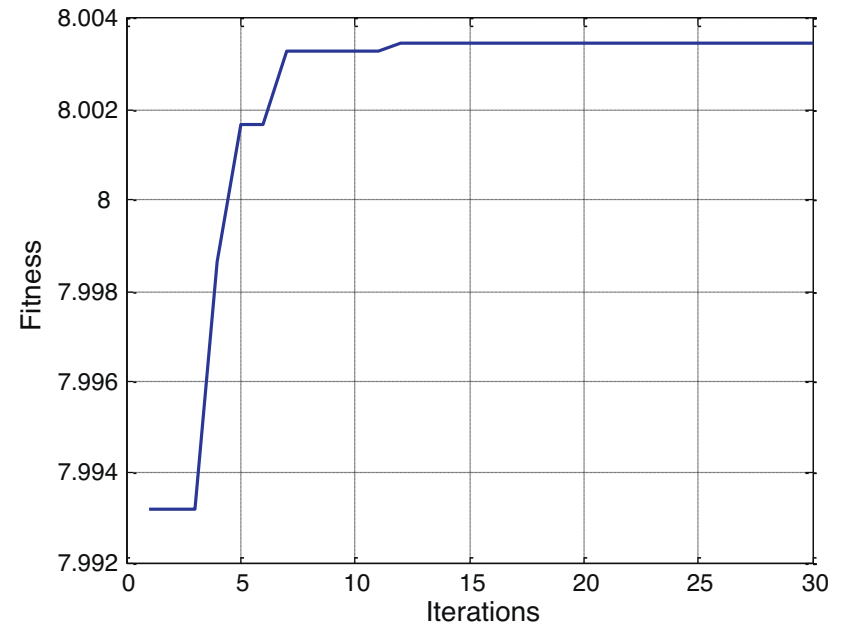

(b)

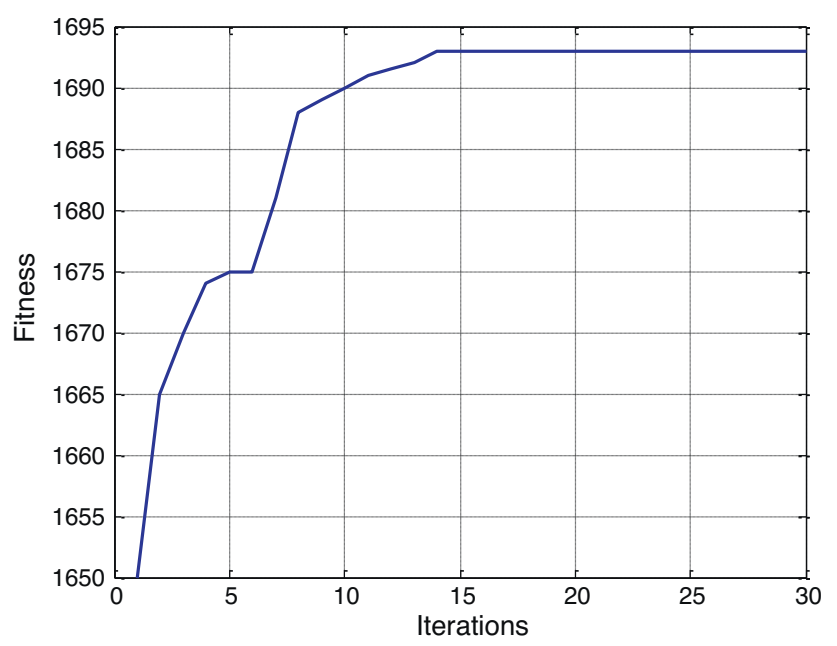

(d)

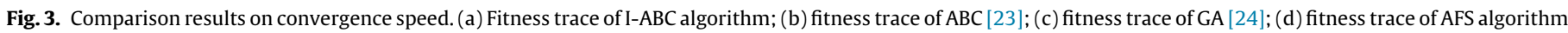
[25].

the optimal threshold value. Fig. 3(d) shows AFS algorithm converges at the 14 th iteration.

(c) Comparison of segmentation performance

In image segmentation applications, there is no global objective criterion to evaluate the quality of the image segmentation methods. But, we can know the desired segmentation results that are constructed by ground truth or human-segmented image. The approach adopted in this study is the ground truth obtained by a simulated image. Therefore, the performance of our developed method is evaluated in terms of Pratt's figure of merit (FOM) using simulated speckled image and ground truth image [36]. The exact and right position of the object edges is to be understood in advance to calculate FOM. FOM technique is described by:

$\mathrm{FOM}=\frac{1}{\max \left\{E_{\mathrm{D}}, E_{\mathrm{I}}\right\}} \sum_{i=1}^{E_{\mathrm{D}}} \frac{1}{1+\alpha\left(d_{i}\right)}$

where $E_{\mathrm{D}}$ and $E_{\mathrm{I}}$ are the number of detected and ideal object edge pixels, respectively. $d_{i}$ is the separation distance of the $i$ th detected edge point normal to a line of ideal object points. $\alpha$ is a scaling constant and was set to $\alpha=1 / 9$ for a stronger penalization of misplaced edge points. FOM value $=1$ corresponds to an excellent similarity between the ideal and detected edge pixels and as the deviation of the detected pixels increases, the FOM value approaches zero.

In Table 5, the FOM values are shown to indicate the quality of the segmentations results by the four methods. According to the FOM results, the proposed edge detector method gives segmentations of high quality and with better results than the other methods for three test image.

\section{Comparison of state-of-the-art machine learning algorithms}

To verify the performance of the proposed segmentation method, well-known real SAR images are used for the experiment. Our segmentation model is tested and compared with the ROEWA [7], MROEWA [8], MRGoA [2], EB-MRGoA [5], IRGS [16] C-MLL [16]

Table 5

FOM results of synthetic and real SAR images for our proposed, ABC, GA and AFS.

\begin{tabular}{lllll}
\hline Image & Our proposed & ABC & GA & AFS \\
\hline Fig. 2(a) & 0.8124 & 0.8243 & 0.4941 & 0.5024 \\
Fig. 2(b) & 0.8274 & 0.8238 & 0.3249 & 0.3056 \\
Fig. 2(c) & 0.8627 & 0.4861 & 0.2543 & 0.2147 \\
Fig. 2(d) & 0.7641 & 0.7259 & 0.2413 & 0.3148 \\
\hline
\end{tabular}


and CHUMSIS [10] models in terms of segmentation result, segmentation accuracy and CPU time.

The first SAR image is a Ku-band $(15 \mathrm{GHz})$ SAR image of the area of China Lake Airport in California [37]. Fig. 4(a) shows the original image. This image has three texture classes: runway (dark region), buildings (bright texture region), and farm (gray texture region). Fig. 4(b) shows the segmentation result obtained by the proposed method. Since our proposed method based on both co-occurrence and brightness features, it can successfully distinguish texture classes. Moreover, the proposed method can extract small runway tributaries. There are only some small segments in the farm area. Our proposed method can separate the runway with the dark area of the farm. Fig. 4(c) shows the segmentation result of the ROEWA method with the empirically chosen threshold value, $T=75$ [7]. This method is dependent on the selected threshold value. Therefore, ROEWA cannot obtain important region edges such as runway and farm regions. Also, it performs over-segmentation in farm region of image. Fig. 4(d) shows the segmentation result obtained by MROEWA method [8]. The region edges detected by the MROEWA method suffer from multiplicative speckle noise. For another thing, this method cannot distinguish the runway with the inner area of the farm and buildings and it cannot divide the runway region with the dark area of the farm texture. It performs over-segmentation in farm regions. Fig. 4(e) shows the segmentation result of the CHUMSIS method [10]. This method can segment three texture regions. The boundaries between different texture regions have been accurately detected. But, CHUMSIS method cannot extract small runway tributaries. This algorithm has two major problems [10]: (1) CHUMSIS uses a deterministic merging process, and (2) the accuracy of super pixels produced by the preprocessing step will also influence the final segmentation. This preprocessing step increases the computational time.
The second real SAR image segmentation is shown in Fig. 5. Fig. 5(a) displays the original SAR image of Piers along the Washington Channel [37]. Fig. 5(b) shows the segmentation result obtained by the proposed neutrosophic method. The boundaries between all piers have been accurately located and detected. The other coastline of channel accurately is detected. Moreover, the roads between buildings and piers have also been exactly segmented out. Fig. 5(c)-(e) shows the segmentation results by the MRGoA [2], EBMRGoA [5] and MROEWA [8] methods, respectively. Fig. 5(c)-(e) clearly shows that extracted piers edges contain noise. Methods perform over-segmentation in the inner regions of piers. Also, the other coastline of channel cannot detect. Because MRGoA, EB-MRGoA and MROEWA methods have weakness against multiplicative speckle noise, segmentation results of them suffer from noise and thin object boundaries. Compared with the other ones, the segmented results obtained by the proposed method have better visual effect.

Fig. 6 shows the segmentation results for a Ku-band $(15 \mathrm{GHz})$ real SAR image in the area of Rio Grande River near Albuquerque [37]. Fig. 6(a) displays the original SAR image. This real SAR image has non-uniform histogram distribution and three texture classes: river (dark region), forest, and farm. Fig. 6(b) displays the segmentation result obtained by applying the proposed method. It detects clear and connected region boundaries. Since the proposed neutrosophic domain information with the histogram features of co-occurrence matrix of the SAR image, two bridges which are difficult to find on the river almost segmented. Fig. 6(c)-(e) show segmentation results obtained by C-MLL, IRGS and CHUMSIS [10], respectively. Both C-MLL and IRGS methods give similar segmentation results. C-MLL and IRGS can only extract main boundaries of three texture classes in image. Since C-MLL and IRGS methods depend on the brightness of pixels, both of them do not work well. When the SAR images have a non-uniform histogram distribution,

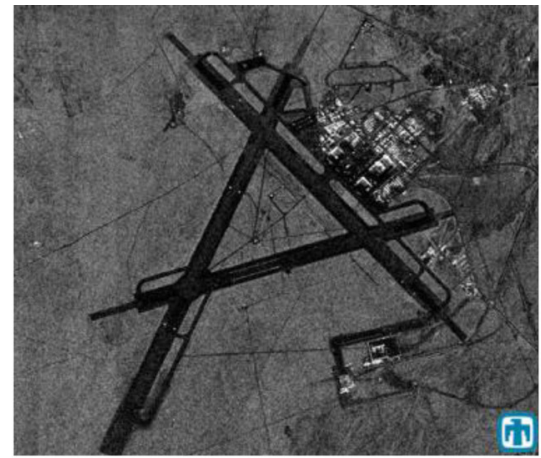

(a)

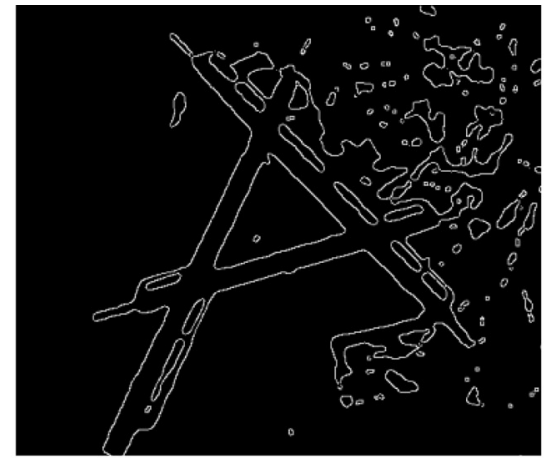

(b)

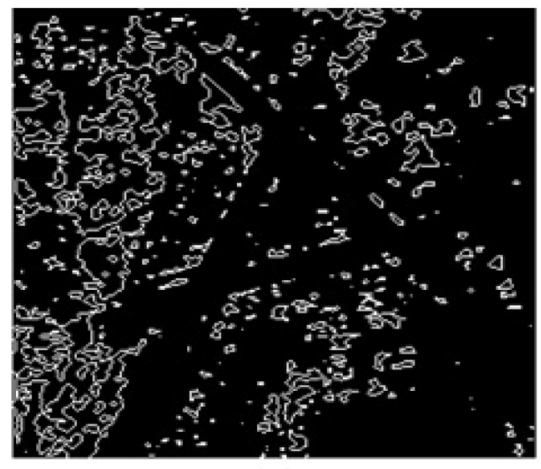

(c)

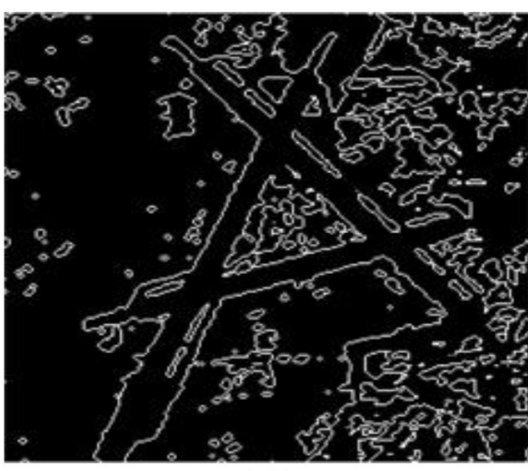

(d)

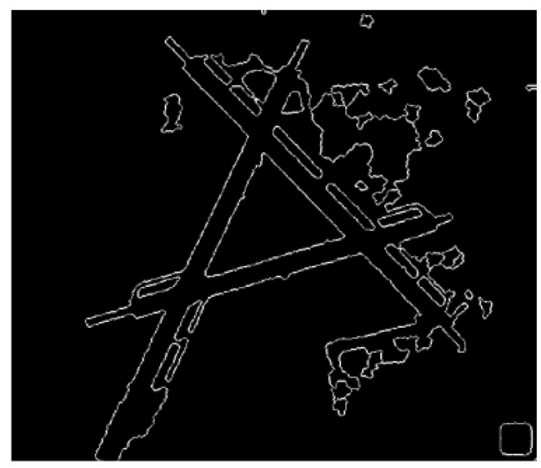

(e)

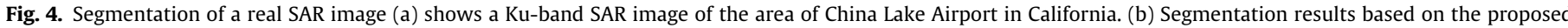

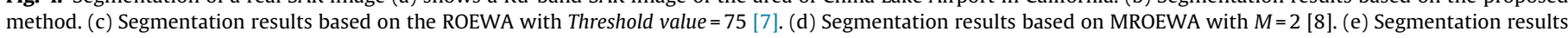
based on the CHUMSIS method [10]. 


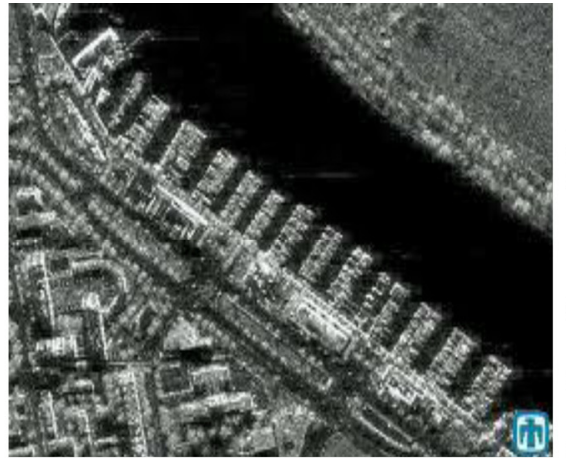

(a)

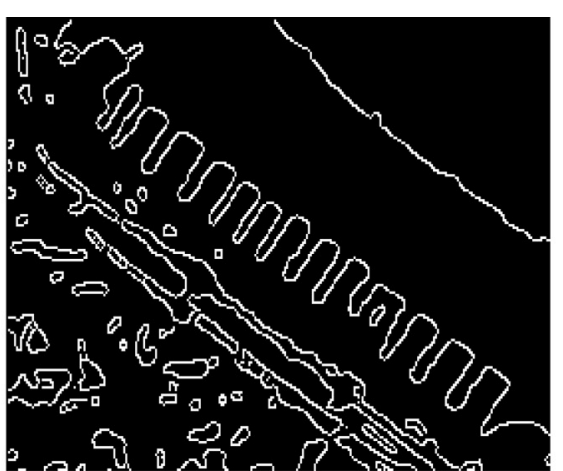

(b)

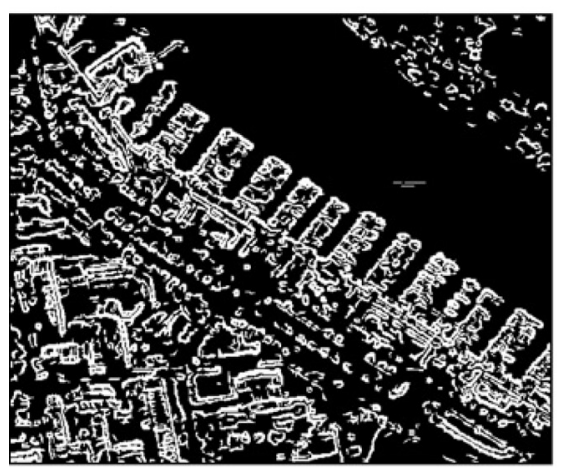

(c)

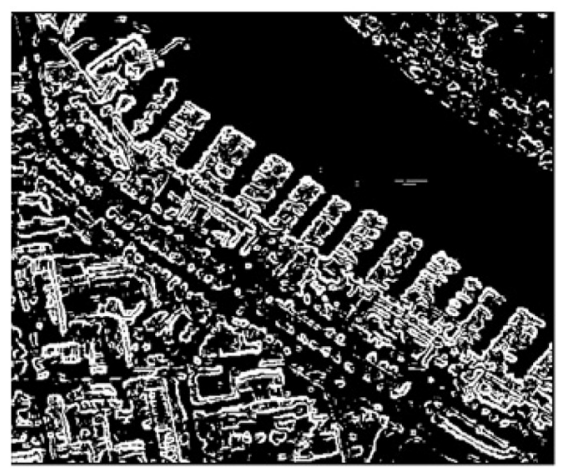

(d)

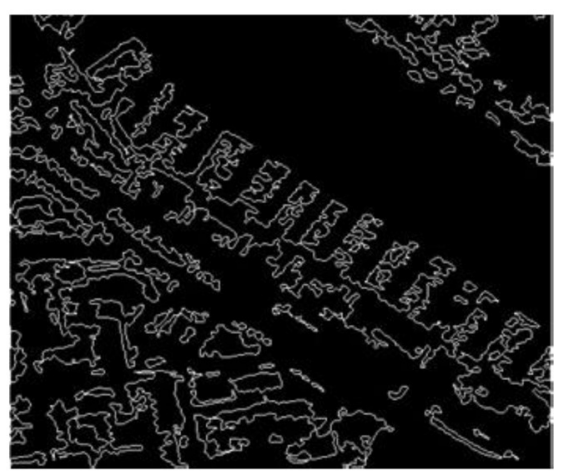

(e)

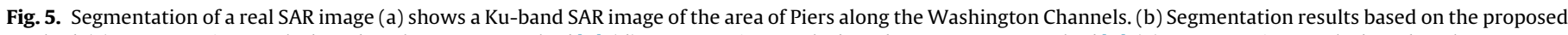

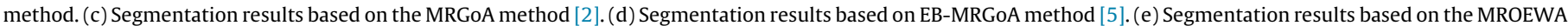
$M=2$ method [8].

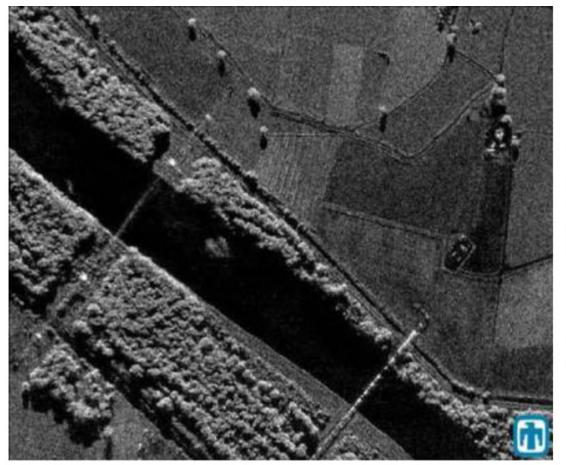

(a)

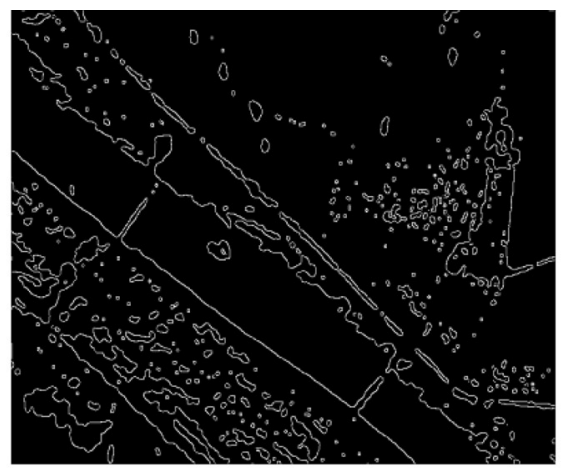

(b)

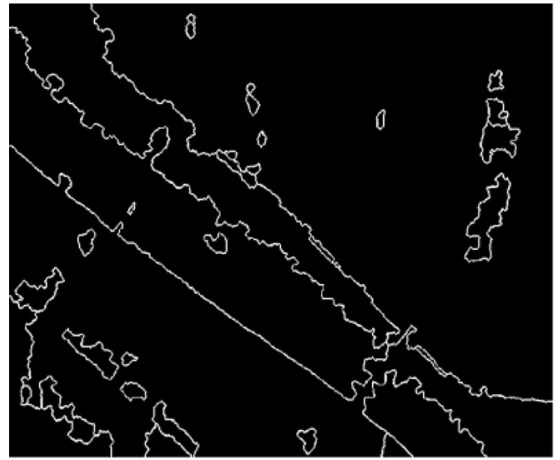

(c)

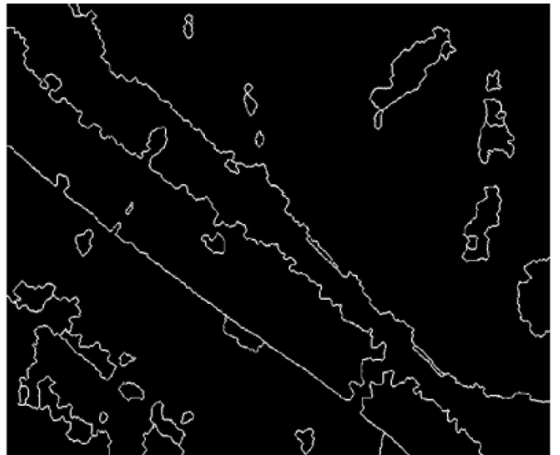

(d)

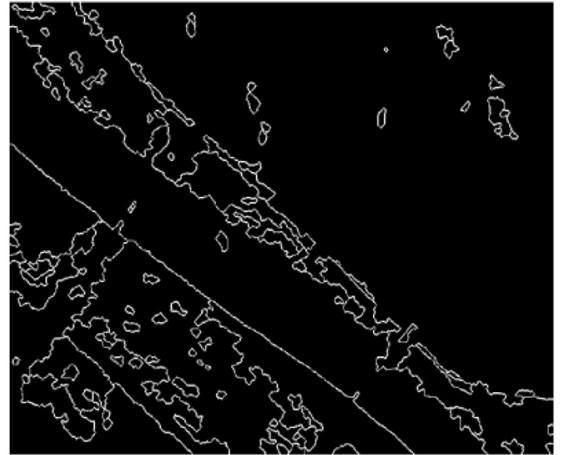

(e)

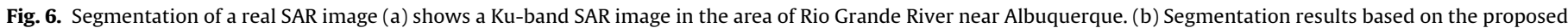

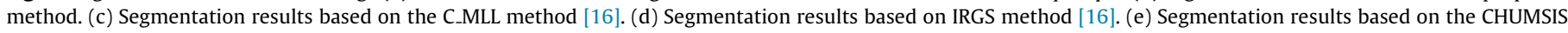
method [10]. 
Table 6

Comparison of the average running time of the algorithms.

\begin{tabular}{|c|c|c|c|c|c|}
\hline & C-MLL & IRGS & CHUMSIS & & The proposed method \\
\hline Running time for preprocessing (s) & & & 42.64 & & \\
\hline Running time for & & & Running time for super-pixel context (s) & 24.08 & \\
\hline segmentation & 4252.77 & 1846.88 & Running time for CMS (s) & 12.46 & 2.55 \\
\hline algorithm (s) & & & Running time for FMS (s) & 30.23 & \\
\hline Total running time $(\mathrm{s})$ & 4295.41 & 1889.53 & 109.42 & & 2.55 \\
\hline
\end{tabular}

Table 7

FOM results of real SAR image for our proposed, ROEWA, MROEWA and CHUMSIS.

\begin{tabular}{lllll}
\hline Image & Our proposed & ROEWA & MROEWA & CHUMSIS \\
\hline Fig. 4(a) & 0.4872 & 0.2146 & 0.4275 & 0.6427
\end{tabular}

\section{Table 8}

FOM results of real SAR image for our proposed, MRGoA, EB-MRGoA and MROEWA.

\begin{tabular}{lllll}
\hline Image & Our proposed & MRGoA & EB-MRGoA & MROEWA \\
\hline Fig. 5(a) & 0.5247 & 0.4156 & 0.4172 & 0.4214 \\
\hline
\end{tabular}

the models of C-MLL and IRGS cannot effectively obtain the super-pixels and obtain super-pixels of different texture classes. Therefore, these methods fail to for Fig. 6(a) image. Since the proposed method based on two-dimensional gray entropy and neutrosophic set, optimal entropic threshold value are obtained using I-ABC algorithm. Because, two-dimensional entropy function is robustness against speckle noise. CHUMSIS method accurately obtains the three texture regions. But, it cannot find the two bridges on the river. Also, CHUMSIS has very long running time.

\section{(a) Comparison of segmentation time}

Comparison of the average running time for the C-MLL, IRGS, CHUMSIS and our proposed method is listed in Table 6. The results of C-MLL, IRGS and CHUMSIS are obtained from the original paper [10]. From Table 6, we can see that the proposed algorithm is much faster than C-MLL, IRGS and CHUMSIS. Also, it should be noted that our method is easier to implement and has much lower time complexity. While C-MLL and IRGS methods need iterative optimization process to calculate the label of all super-pixels, CHUMSIS uses a deterministic optimization strategy. Although CHUMSIS model can detect the desired object boundaries, it has high computing time. Because of that it is not suitable for real time SAR image applications. The proposed method uses I-ABC optimization algorithm for estimate the optimal threshold value. Average running time results show that I-ABC algorithm is superior to SAR image segmentation, and it is a successful method in detecting the object boundaries, with the advantages of fast convergence and robustness. Also, the proposed method can meet the requirements of the real time SAR image segmentation.

(b) Comparison of segmentation performance

In Tables 7-9 the FOM values are shown to indicate the quality of the segmentations results by the four methods. According to the FOM results, the developed method gives segmentations with better results than the other state-of-the-art methods for many real SAR images.

Table 9

FOM results of real SAR image for our proposed, C-MLL, IRGS and CHUMSIS.

\begin{tabular}{lllll}
\hline Image & Our proposed & C-MLL & IRGS & CHUMSIS \\
\hline Fig. 6(a) & 0.4652 & 0.3325 & 0.3140 & 0.4597 \\
\hline
\end{tabular}

\section{Conclusion}

In this paper, we proposed a fast and robust segmentation method to solve the multi-class SAR image segmentation problem. A SAR image segmentation algorithm must obtain both right and smooth object boundaries. In order to ensure two major purposes mentioned above, a new hybrid feature extracting model based on Neutrosophic set and co-occurrence matrix is presented in this paper. Since original $A B C$ algorithm is weak mathematically, we used the I-ABC algorithm. We combine neutrosophic domain features with the histogram features of co-occurrence matrix of the SAR image. This model assures reasonable foraging guide for I-ABC algorithm. In this way, robust and fast I-ABC algorithm with an efficient fitness function can be obtained. Two-dimensional gray entropy function obtained from the co-occurrence matrix is used as the fitness function of I-ABC algorithm. The proposed method regards threshold estimation as a search process and employs I$\mathrm{ABC}$ algorithm to optimize the two-dimensional entropy function. I-ABC algorithm provides a fast convergence speed. Therefore, our algorithm can be used in real time SAR image processing. Additionally, the proposed method gives right and noise-free object edges. The segmentation results of synthetic and real SAR images show the speediness and efficiency of our proposed method. Experimental results show that the proposed method effectively improves the segmentation accuracy of the SAR image while reducing the influence of multiplicative speckle noise.

\section{References}

[1] G. Gao, L. Zhao, J. Zhang, D. Zhou, J. Huang, A segmentation algorithm for SAR images based on the anisotropic heat diffusion equation, Pattern Recogn. 41 (10) (2008) 3035-3043.

[2] Z. Bai, P. He, An improved ratio edge detector for target detection in SAR images, in: Proc. IEEE Int. Conf. Neural Netw. Signal Process., 2003, pp. 982-985.

[3] H. Sui, F. Peng, C. Xu, K. Sun, J. Gong, GPU-accelerated MRF segmentation algorithm for SAR images, Comput. Geosci. 43 (June) (2012) 159-166.

[4] M. Li, Y. Wu, Q. Zhang, SAR image segmentation based on mixture context and wavelet hidden-class-label Markov random field, Comput. Math. Appl. 57 (March (6)) (2009) 961-969.

[5] J. Jennifer Ranjani, S.J. Thiruvengadam, Entropy based segmentation for visual content description in CBIR using SAR images, in: Proc. Int. Conf. Content Based Image Retrieval, 2008, pp. 139-142.

[6] Y. Wu, P. Zhang, M. Li, Q. Zhang, F. Wang, L. Jia, SAR image multiclass segmentation using a multiscale and multidirection triplet Markov fields model in nonsubsampled contourlet transform domain, Inform. Fusion 14 (4) (2013) 441-449.

[7] J.J. Ranjani, M. Gokila, S.J. Thiruvengadam, Edge detection in speckled SAR images with improved ROEWA, in: IEEE Proc. Sixth Indian Conf. Comput., Vision, Graph. Image Process., 2008, pp. 644-649.

[8] J.J. Ranjani, S.J. Thiruvengadam, Fast threshold selection algorithm for segmentation of synthetic aperture radar images, IET Radar, Sonar Navigation 6 (8) (2012) 788-795.

[9] R. Jobanputra, D.A. Clausi, Preserving boundaries for image texture segmentation using grey level co-occurring probabilities, Pattern Recogn. 39 (2006) 234-245.

[10] H. Yu, X. Zhang, S. Wang, B. Hou, Context-based hierarchical unequal merging for SAR image segmentation, IEEE Trans. Geosci. Remote Sens. 51 (February (2)) (2013) 995-1009.

[11] M.S. Horritt, A statistical active contour model for SAR image segmentation, Image Vision Comput. 17 (3/4) (1999) 213-224.

[12] X.J. Yuan, N. Situ, G. Zouridakis, A narrow band graph partitioning method for skin lesion segmentation, Pattern Recogn. 42 (2009) 1017-1028.

[13] P. Zhong, R.S. Wang, A multiple conditional random fields ensemble mode for urban area detection in remote sensing optical images, IEEE Trans. Geosci. Remote Sens. 45 (12) (2007) 3978-3988. 
[14] J. Feng, Z. Cao, Y. Pi, Multiphase SAR image segmentation with $\mathrm{G}^{0}$-statisticalmodel-based active contours, IEEE Trans. Geosci. Remote Sens. 51 (7) (2013) 4190-4199.

[15] Y. Wu, K. Ji, W. Yu, Y. Su, Region-based classification of polarimetric SAR images using Wishart MRF, IEEE Geosci. Remote Sens. Lett. 5 (October (4)) (2008) 668-672.

[16] Q. Yu, D.A. Clausi, IRGS: image segmentation using edge penalties and region growing, IEEE Trans. Pattern Anal. Mach. Intell. 30 (December (12)) (2008) 2126-2139.

[17] M. Sezgin, B. Sankur, Survey over image thresholding techniques and quantitative performance evaluation, J. Electron. Imaging 13 (1) (2001) $146-168$.

[18] G. Li, P. Niu, X. Xiao, Development and investigation of efficient artificial bee colony algorithm for numerical function optimization, Appl. Soft Comput. 12 (1) (2012) 320-332.

[19] F. Smarandache, A Unifying Field in Logics Neutrosophic Logic. Neutrosophy, Neutrosophic Set, Neutrosophic Probability, third ed., American Research Press, 2003.

[20] Y. Guo, H.D. Cheng, A new neutrosophic approach to image segmentation, Pattern Recogn. 42 (2009) 587-595.

[21] D. Karaboga, An Idea Based on Bee Swarm for Numerical Optimization, Technical Report-TR06, October, 2005

[22] D. Karaboga, B. Basturk, A powerful and efficient algorithm for numerical function optimization: artificial bee colony (ABC) algorithm, J. Global Optim. 39 (3) (2007) 459-471.

[23] M. Ma, J. Liang, M. Guo, Y. Fan, Y. Yin, SAR image segmentation based on Artificial Bee Colony algorithm, Appl. Soft Comput. 11 (8) (2011) 5205-5214.

[24] G. Chen, H.F. Zuo, 2-D maximum entropy method of image segmentation based on genetic algorithm, J. Comput.-Aided Des. Comput. Graph. 14 (6) (2002) 530-534.

[25] Z. Pan, Y.Q. Wu, The two-dimensional Otsu thresholding based on fish-swarm algorithm, Acta Optica Sinica 29 (8) (2009) 2115-2121.
[26] C.-T. Li, R. Chiao, Multiresolution genetic clustering algorithm for texture segmentation, Image Vision Comput. 21 (11) (2003) 955-966.

[27] A. Sengur, Y. Guo, Color texture image segmentation based on neutrosophic set and wavelet transformation, Comput. Vision Image Understand. 115 (2011) 1134-1144.

[28] J. Mohan, V. Krishnaveni, Y. Guo, MRI denoising using nonlocal neutrosophic set approach of Wiener filtering, Biomed. Signal Process. Control 8 (6) (2013) 779-791.

[29] Y. Guo, H.D. Cheng, A new neutrosophic approach to image denoising, New Math. Natural Comput. 5 (3) (2009) 653-662.

[30] K. Pawalai, Chun Che Fung, Binary classification using ensemble neural networks and interval neutrosophic sets, Neurocomputing 72 (13-15) (2009) 2845-2856.

[31] F. Gao, F.-x. Fei, Y.-f. Deng, Y.-b. Qi, B. Ilangko, A novel non-Lyapunov approach through artificial bee colony algorithm for detecting unstable periodic orbits with high orders, Expert Syst. Appl. 39 (16) (2012) 12389-12397.

[32] M.-H. Horng, Multilevel thresholding selection based on the artificial bee colony algorithm for image segmentation, Expert Syst. Appl. 38 (11) (2011) 13785-13791.

[33] K. Hanbay, M.F. Talu, A. Karci, Segmentation of color texture images with artificial bee colony algorithm and wavelet transform, in: Proc. 20th IEEE Signal Process. Commun. Appl. Conf. (SIU), 2012, pp. 1-4.

[34] Z. Hui, J.E. Fritts, S.A. Goldman, Image segmentation evaluation: a survey of unsupervised methods, Comput. Vision Image Understand. 110 (2) (2008) 260-280.

[35] H.Q. Zhu, Segmentation of blood vessels in retinal images using 2D entropies of gray level-gradient cooccurrence matrix, in: Proceedings of IEEE International Conference on Acoustics, Speech, and Signal Processing, 2004 (ICASSP '04), 2004, iii, 509-512.

[36] I.E. Abdou, W.K. Pratt, Quantitative design and evaluation of enhancement/thresholding edge detectors, Proc. IEEE 67 (5) (1979) 753-763, http://www.sandia.gov/radar/imageryku.html 